\title{
A new method for calculating energy of matter
}

Qing Li*

*ShiJiaZhuang Traditional Chinese Hospital, ShiJiaZhuang, China

\begin{abstract}
An approximate calculation of the spatial characteristics on finite range is required, so one quantitative continuum represents the accumulation of infinite great quantities is artificially divided it into smaller and camparable parts in which calculus operation can be applied .This operation is defined as Theorem 1 in which infinity is not involved, there is a camparable finity is constantly (forever) approaching and not reaching infinity, and only staying within a finite range. Theorem 1 can exist in this paper as a new mathematical basis for physics. Because the essence of all physical quantities is size comparison, and the size comparison relation of matter can only be space/time, so relation formula space/time is the only expression of the concept of matter, all physical quantities are applicable to this expression, each different physical quantity is a multidimensional representation of this expression. A new mass energy formula is aslo derived from this paper.
\end{abstract}

Key words : mass energy formula, space/time, the camparable finity

\section{Introduction}

As mentioned earlier, Axiom 3 [1] is a modification of Axiom 1 [2], so in Axiom 1, the notion that space is a non-continuum composed of an infinite number of 0 points (singularities) is no longer applied. In Axiom 3, space is one quantitative continuum, which means that an infinite small quantity does not exist. Instead, one quantitative continuum represents the accumulation of infinite many quantities. Apart from Axioms 1 and 3, there is no third spatial feature, so one reader may ask, in common sense, why are we able to apply calculus to describe the spatial features we see? Before we expand on this subject in detail, it is necessary to explain the basic principles of calculus operation. The calculus operation is based on Axiom 2[1], where space is an continuum consisting of infinitesimal components in which the different parts can compare in size with each other. In order to justify itself, Axiom 2 gives infinitesimal an abstract concept, that is, a minimun concept, which is infinitely close but cannot be reached. It is known from Axiom 1 and 3, if the space is a continuum, it is one quantitative and there is no infinitesimal , and if the space is composed of infinitesimal so it is a non-continuum, there is no third Axiom compromise between these two concepts. So the calculus operation based on Axiom 2 does not make sense for infinitesimal and infinite great. Because in our daily life, an approximate calculation of the spatial characteristics is required, so the calculus operation is essential and is needed to redefine in order to better use this calculation.

\section{The mathematical meaning of Theorem 1}

Preliminaries The one quantitative continuum of Axiom 3 is a quantitative continuum that extends to an infinite distance and cannot be divided into smaller parts, that is, its parts cannot be compared in size. But in everyday life, for computing applications, this one quantitative continuum is artificially divided it into smaller parts, The prerequisite for it is that it must be defined as follows: 
Given a divided parts, let's call it $a$, it's defined as a finite quantities, and every quantities different from it is a finite quantities, let's call it $b$, So $b / a=$ finite, and since both $a$ and $b$ are finite, so in this divisible system, there is neither a maximum nor a minimum. Since infinity is not involved, $b$ is constantly (forever) approaching and not reaching infinity, but only staying within a finite range. Here decimal points and fractions make sense, and irrational Numbers make sense (the circle to the decimal point can only stay within a finite range) and dimensions of space are also comparable in size. This redefined calculus is called as Theorem 1.

Now the limit of the function in Theorem 1 is defined. Cartesian coordinates also can be applied to Theorem 1.

(1) The limit of the function as the independent variable approaches a specific finite value.

Definition 1 Assuming that the function is defined in some region at the point $\mathrm{x}_{0}$ of $\mathrm{f}(\mathrm{x})$, if there is constant $\mathrm{A}$, for any given positive $\varepsilon$ (no matter how small, but within a finite range), there will always be positive $\delta$, such that when $\mathrm{x}$ satisfies the inequality $0<\left|\mathrm{x}-\mathrm{x}_{0}\right|<\delta$, the corresponding value of the function will satisfy the inequality $|\mathrm{f}(\mathrm{x})-\mathrm{A}|<\varepsilon$, So the constant $\mathrm{A}$ is called the limit of the function $\mathrm{f}(\mathrm{x})$. Being written for:

$$
\lim _{x \rightarrow x_{0}} f(x)=A
$$

In contrast to the previous calculus definitions, $\mathrm{x}$ and $\mathrm{x}_{0}$ have different ranges of definition, for example, $\mathrm{x}$ is constantly (forever) approaching and not reaching $\mathrm{x}_{0}$, and $\mathrm{x}$ and $\mathrm{x}_{0}$ can only stay within a limited range.

(2) The limit of the function when the independent variable approaches infinite but only stays within a finite range.

Definition 2 Suppose the function is defined in $f(x)$ when $|x|$ is greater than the positive number.,If there is A constant, for any given positive number $\varepsilon$ (no matter how small it is, but it must be in a finite range), there will always be positive $\eta$, so that when $x$ satisfies the inequality $\mid x$ $P \eta$, the corresponding function value satisfies the inequality $|\mathrm{f}(\mathrm{x})-\mathrm{A}|<\varepsilon$, So the constant $\mathrm{A}$ is called the limit of the function $\mathrm{f}(\mathrm{x})$. Being written for:

$$
\lim _{x \rightarrow \infty} f(x)=A
$$

(3)Now the derivative of the function is defined in Theorem 1. Cartesian coordinates can be also applyied to Theorem 1.

Definition 3 Let function $\mathrm{y}=\mathrm{f}(\mathrm{x})$ is defined in a certain region of point $\mathrm{x}$ (limited range), When independent variable $\mathrm{x}$ gets increment $\triangle \mathrm{x}$ (point $\mathrm{x}_{0}+\triangle \mathrm{x}$ is still in the neighborhood), dependent variable gets increment $\triangle \mathrm{y}=\mathrm{f}\left(\mathrm{x}_{0}+\triangle \mathrm{x}\right)-\mathrm{f}\left(\mathrm{x}_{0}\right)$ correspondingly. If the limit of the ratio of $\Delta \mathrm{y}$ to $\triangle \mathrm{x}$ exists when $\triangle \mathrm{x} \rightarrow 0$, then the function $\mathrm{y}=\mathrm{f}(\mathrm{x})$ is differentiable at the point $\mathrm{x}_{0}$, and the limit is the derivative of the function $y=f(x)$ at the point $x_{0}$, which is called $f^{\prime}\left(x_{0}\right)$, that is,

$$
f^{\prime}\left(x_{0}\right)=\lim _{\Delta x \rightarrow 0} \Delta y / \Delta x=\lim _{\Delta x \rightarrow 0} f\left(x_{0}+\Delta x\right)-f\left(x_{0}\right) / \Delta x
$$

Or it can be aslo called $y^{\prime} \mid x=x_{0}$, or $d y / d x \mid x=x_{0}$

The other operations of calculus are given in turn by the two definitions above. Note that the symbols $x \rightarrow 0$ 及 $x \rightarrow \infty$ indicate that the independent variable $x$ approaches infinitely (continuously, forever) and fails to reach infinitesimal or infinity, but only stays within a finite 
range.

\section{The physical meanings of Theorem 1}

\section{Main results}

Theorem 1 gives the geometric characteristics of finite range of time and space. It also provides a mathematical basis for us to study the relations and characteristics of physical quantities in a limited range. Some readers will ask: How does the new mathematical model describe the characteristics of each physical quantity? Further, what is the meaning of each quantity? That is, what is the essence of matter? This article focuses on this concept. In our daily life, the matter is described as solid, liquid and gas with mass and spatial extensibility and here the concept of matter can be separated from the concept of space and time, even though matter is spatially extensive. So what is the nature of matter, how is the concept of matter defined. Einstein once said: 'If space and time disappear, matter will not exist'. This sentence deeply defines the concept of matter, that is, the essence of matter is time and space. Such a definition goes against our usual understanding of matter because matter with mass is different from the vacuum we see in common. Further, here the density of matter is different, that is, different substances with the same volume have different masses.In view of the fact that the essence of matter is time and space, how to use the geometric characteristics of time and space to express the different density of matter? This paper focuses on this concept.

Before discussing the concrete meaning of matter, the finite range of space-time characteristics will be restated to make our understanding of physical quantities in the new state clearer.

(1)Cartesian coordinates can also apply to Theorem 1 [3]. In a finite range, all spatiotemporal functions describing the motion of lines and arbitrary curves are applicable to Theorem 1 . The Cartesian coordinate system, in which space is ordinate and time is abscissa, is applicable to describe all the characteristics of physical quantities in the Theorem 1. In Theorem 1, space and time is a continuum with no maximum or minimum but comparable size, and in a finite range, a given length can be arbitrarily and forever divided into smaller quantities.

(2)Since Theorem 1 is the approximate calculation of the measurement values of Axiom 1 and 3 in a finite range, it follows the inertial system principle of Axiom 1 and the universal velocity invariant principle. In Axiom 1, the universal velocity invariant principle means that each velocity is itself and not any other velocity, that each velocity is invariant with respect to the other velocities. The inertial system principle of Axiom 1 is defined that cartesian coordinate system cannot describe the distribution of space-time variables, that is, all space-time quantities at rest relative to a coordinate system do not exist (such as $K$ or $K^{\prime}$ ). A particular quantity represents only one state, so the motion states of all different quantities are absolute and the comparison of the motion states of two quantities is also absolute. On the basis of these two principles, the minimum state in Theorem 1 (equivalent to the static state of cartesian inertial system with velocity of 0 ) is defined as an infinite (continuously, forever) approaching and not reaching infinitesimal, but can only stay in a finite range. These two principles also determine that space and time are dependent of each other in Theorem 1. Although space and time in Axioms 1 and 3 are single dimensional, that is, the magnitude of space and time is determined by one-dimensional unit values, rather than by the ratio of space and time (velocity) of multiple dimensions. However, the geometric segments of space and time in Theorem 1 are artificially divided into finite parts, and the two cannot be 
fused into a single dimension (in Axiom 3, time and space are fused to a quantity at an infinite distance). Therefore, space and time in Theorem 1 are multi-dimensional. For example, in a $3 \mathrm{~m} / \mathrm{s}$ speed event, $3 \mathrm{~m}$ and $1 \mathrm{~s}$ are two events, but they are dependent of each other.Since the essence of matter is time and space, all physical quantities, such as mass, energy, temperature, charge, etc., are the same concept in Theorem 1.

(3)The meanings and definitions of substances(matter)

Now the concept of matter is illustrated. As mentioned before, the material is the nature of time and space, and there is no third things exist, and because the material of various fundamental physical quantities have size comparison, such as for mass of a physical quantity, the same volume of mass is different, therefore, the size comparison relation of matter can only be space/time $(s / t)$, it is the only expression of the concept of matter, all physical quantities are applicable to this expression, each different physical quantity is a multi-dimensional representation of this expression.

Now a detail mathematical expression for this definition of matter is given .Cartesian coordinates with $\mathrm{f}(\mathrm{t})$ in space and $\mathrm{t}$ in time are suitable for describing this expression.

Definition 4 Assuming that spatial function $y=f(t)$ in space-time function be bounded in the finite range of $[a, b]$, insert some points in $[a, b]$,

$\mathrm{a}=\mathrm{t}_{0}<\mathrm{t}_{1}<\mathrm{t}_{2}<\cdots \cdots \cdot \mathrm{t}_{\mathrm{n}-1}<\mathrm{t}_{\mathrm{n}}=\mathrm{b}$

Divide the interval $[a, b]$ into $n$ smaller interval,

$\left[\mathrm{t}_{0}, \cdots \cdots \mathrm{t}_{1}\right],\left[\mathrm{t}_{1}, \mathrm{t}_{2}\right], \cdots \cdots,\left[\mathrm{t}_{\mathrm{n}-1}, \mathrm{t}_{\mathrm{n}}\right]$,

The length of each small interval in turn is

$\triangle \mathrm{t}_{1}=\mathrm{t}_{1}-\mathrm{t}_{0}, \quad \triangle \mathrm{t}_{2}=\mathrm{t}_{2}-\mathrm{t}_{1}, \cdots \cdots, \Delta \mathrm{t}_{\mathrm{n}}=\mathrm{t}_{\mathrm{n}}-\mathrm{t}_{\mathrm{n}-1}$,

Take a point $\xi_{\mathrm{i}}\left(\mathrm{t}_{\mathrm{i}-1}<\xi_{\mathrm{i}} \leqslant \mathrm{t}_{\mathrm{i}}\right)$ between each small interval $\left[\mathrm{t}_{\mathrm{i}-1}, \mathrm{t}_{\mathrm{i}}\right]$, Taking the product of the function value $f\left(\xi_{i}\right)$ with the small interval length $\triangle t_{i}, f\left(\xi_{i}\right) \triangle t_{i}(i=1,2, \cdots \cdots, n)$, and make the sum

$$
S=\sum_{i=1}^{n} f\left(\xi_{i}\right) \triangle t_{i}
$$

Denoted $\lambda=\max \left\{\triangle \mathrm{t}_{1}, \triangle \mathrm{t}_{2}, \cdots \cdots, \triangle \mathrm{t}_{\mathrm{n}}\right\}$, If the limit of the sum always exists when $\lambda \rightarrow 0$ (which means that the infinite (constantly, forever) is close and not reach infinitesimal, and can only stay in a finite range), then the limit is called the definite integral of the function $f(t)$ on the interval [a,b],Written for,

$$
\int_{a}^{b} f(t) d t
$$

Namely

$$
\int_{a}^{b} f(t) d t=\lim _{\lambda \rightarrow 0} \sum_{i=1}^{n} f\left(\xi_{i}\right) \triangle t_{i}
$$

Where $f(t)$ is called the or the space-time derivative.

So far the exact definition of matter has been given, which is the definite integral of space over time. There are four points to this definition. (1) Space and time are dependent of each other in Theorem 1, so we use the ratio of space to time (velocity) of the two dimensions to determine the magnitude of the physical quantity. Space and time are still two different events. For example, in the event of motion of $1 \mathrm{~m} / \mathrm{s}, 1 \mathrm{~m}$ and $1 \mathrm{~s}$ are two different events, not one event (where $1 \mathrm{~m}$ and 1 
$\mathrm{s}$ are equivalent here), but both relate to each other. (2) According to the definition of Theorem 1, the exact definition of $t_{n}$ point is the two endpoints of finite length, not 0 point. Nor $\xi$ is the point 0 , it is finite length quantities infinitely close to infinitesimall. (3)Definite integral is the simplest mathematical expression to describe the relationship between space and time. Other more complex space-time relations can be described by partial derivatives, multiple integrals, curve integrals, surface integrals, etc. These relations do not affect the basic definition of matter, namely, space/time. (4)Some readers may ask, how does this definition define the sizes for all physical quantities? For example, Concerning the mass, a physical quantity, if you have different masses in the same volume, how do you define its size? From the definition we can see that different matter of the same volume have different masses because the different matter of the same volume in space have different time values, so from this point of view, the rigid body matter that we observe in space is not only spatially extensional, but also temporally extensional.

Some readers may ask, the definition of speed is also space/time, so how does it differ from other physical quantities? Since the essence of matter is the integral of space over time, all physical quantities are essentially one concept and have multi-dimensional characteristics. But they are independent of each other in different forms in nature and are different representations of space/time, so they can be transformed into each other in essence. For example, if we talk about the velocity of motion of a particle with mass, we usually say that the mass and velocity of the particle are two different forms. Although the essence of mass and velocity is space/time, they are different representations of space/time and cannot be substituted for each other in forms, and they are only dependent of each other in concept. This definition does not preclude the fact that each of the two quantities has multi-dimensional characteristics.

But the Relativistic equation $E=\mathrm{mc}^{2}$ [4] does not depend on the fact that the mass and velocity of a particle are one concept, but on the notion that they are the different thing. In Relativity, the speed of light is the basis for defining space and time (namely light time and light space). One second is equivalent to 300,000 kilometers, and the speed of light remains constant in any reference inertia, the definition of all velocities depend on the value of the speed of light. Therefore, in a static coordinate system with a velocity of 0 , the time and space values of the speed of light exist as the minimum rest state, where its energy is equal to its mass and both are one concept. And in a coordinate system of motion, energy is not equal to the static mass, and the mass of a substance is not a constant, it varies with the velocity. In the mass energy equation $\mathrm{E}=\mathrm{mc}^{2}$, the energy of $\mathrm{M}$ at rest is given by $\mathrm{mc}^{2}$, and in the cartesian inertial system moving with velocity $v, E=m / \sqrt{ }\left(1-v^{2} / c^{2}\right)$, that is, the value of $m$ increases with the increase of velocity $v$ of the inertial system.However, the above this is a strange conclusion.

This strange conclusion will discard due to the new definition of matter in Theorem 1. Although the essence of matter is space/time and all physical quantities are its manifestation, each physical quantity has different manifestations. For example, mass and velocity are two manifestations . In addition, since Theorem 1 follows the inertial system principle of Axiom 1, that is, space-time quantities at rest with respect to a coordinate system do not exist (for example, $K$ or $K^{\prime}$ ) ,A particular quantity represents only one state, so the motion states of all different quantities are absolute, and the comparison of the motion states of two quantities is also absolute. Therefore, the minimum rest state of Theorem 1 is space $\rightarrow 0 /$ time $\rightarrow \infty$.

So the mass energy equation has a new expression in Theorem 1 where mass and velocity with respect to the minimum state of rest are defined as follows. 
Definition 5 If a given mass is $m_{i}$ and a given velocity is, then the magnitude values of mass and velocity are $s_{i}$ and $h_{i}$ :

$$
S_{1}=\lim _{m 0 \rightarrow 0} m_{1} / m_{0}
$$

and

$$
h_{1}=\lim _{v_{0} \rightarrow 0} v_{1} / v_{0}
$$

Here $\mathrm{M}_{0} \rightarrow 0$ 和 $\mathrm{v}_{0} \rightarrow 0$ represent the minimum rest state of velocity in Theorem 1 , that is, space $\rightarrow 0$ / time $\rightarrow \infty$. Here, space $\rightarrow 0$ and time $\rightarrow \infty$ represent space infinity (continuously and forever) approaching infinitesimally small and unreachable, and time infinity approaching infinitesimally large and unreachable, and can only stay in the finite range.

Newton's physical quantity In Newton's classical physics, each physical quantity is not the same concept, so energy, velocity and mass are three physical quantities, independent of each other. In general, the energy of a matter in physics is defined as work, the magnitude of which is equal to the product of the force and the distance through which the object passes in the direction of the force. Based on the previous definition, we can obtain the relation of mass and energy in Theorem 1, which is shown here

$\mathrm{E}=\mathrm{mv} \mathrm{v}^{2}$

The units of mass are still expressed in kilograms, so the units of energy are still joules ( 1 joule $=1$ kilogram $\times 1$ square meter $/ 1$ square second).

There are aslo two points to emphasize in this equation: (1)If we are talking about the energy of a mass object, and its speed is different, then its energy is different. For example, for a photon, the energy it has is $\mathrm{E}=\mathrm{mc}^{2}$, but for other objects that are not moving at the speed of light, the energy it has is $E=m v^{2}$. (2) The mass of an object is constant. Unlike Relativity, the formula $E=m v^{2}$ remains constant in any inertial system (that is, independent of any inertial system state), so the mass of any substance does not change with its velocity.

Physical quantity in Theorem 1 In Theorem 1, all physical quantities are expressed by the formula space / time, that is, here they have unity, so they are a concept. It can be concluded that if an acceleration has curve or manifold characteristics, the two physical quantities of velocity and mass also have manifold characteristics, so does energy, and so on. So where do the different characteristics of different physical quantities come from? In fact, they are just different forms in nature.

Therefore, the dimension of each physical quantity has specificity, that is, it has a dimensional system different from other physical quantities. For example, for the physical quantity of mass, the comparison of the size of each different mass is only meaningful in its interior, and has nothing to do with the internal size comparison of other physical quantities (such as velocity). This is determined by the nature of Theorem 1 in which there are no maximun and minimum quantities. Therefore, from this point of view, each physical quantity has both unity and difference. This is also determined by the properties of Theorem 1 .

As mentioned earlier, due to the differences of each physical quantity, we cannot directly compare 
the size of two different physical quantities. For example, $1 \mathrm{~g}$ of material cannot be compared with a speed of $3 \mathrm{~m} / \mathrm{s}$. Because of the essential identity of each physical quantity, the comparison of the size of the two physical quantities is artificially defined. For example, in the mass energy equation $\mathrm{E}=\mathrm{mc}^{2}$, this formula can be established in Theorem 1 only if the mass of 1 gram of material is equivalent to the speed of light. It can be seen that in addition to the speed and mass of the two physical quantities, the energy of the third physical quantity is given in this way, and so on, More physical quantities are given. Itt can be known here that the relationship between the physical quantities defined in this way is given as $s=h$ in the above formula. If we define that 1 gram is equivalent to a given $\mathrm{h}_{\mathrm{j}}$ (unit $m / s$, expressed in $\mathrm{k}$ ), then this formula can be written as $\mathrm{E}=\mathrm{mk}^{2}$. In describing the three relationships in Theorem 1, the formula 9 still use the old definitions for measuring their size in formula 8 .

$\mathrm{E}=\mathrm{mk}^{2}$

Or

$\mathrm{E}=\mathrm{m}^{3}=\mathrm{k}^{3}$

There are aslo two points to emphasize in this equation: (1)When we talk about the energy of a moving particle, its velocity and mass are equivalent, so there is no matter with static mass and energy (velocity is 0 ). Similarly, it is meaningless to talk about a motion without mass (mass is 0 ).

(2) When we talk about the energy of a moving particle, the change of velocity means the change of mass, the change of mass means the change of velocity, and these two changes also mean the change of energy. For a substance with a velocity of 0 , its mass and energy are also 0 . (3)In the relativistic mass energy equation, energy and mass are one concept, which is consistent with the concept of Theorem 1. In any case, they are not the same concept as velocity, which is inconsistent with theorem 1. In Theorem 1, the three are one concept. Furthermore, all physical quantities are one concept, that is, they are space /time.

The speed of light is constant In modern physics, each physical quantity is independent of each other, and each physical quantity has its own unit of measurement. However, In Theorem 1, each physical quantity is a concept, so they should have a unified unit of measurement. In formula $9, \mathrm{k}$ is an arbitrary finite value, so it cannot exist as this unified unit. Since the essence of all physical quantities is space / time, Then we take the physical quantity of velocity as the most basic unified unit quantity. In the physical quantity speed, we specify the speed of light as a constant, which has two meanings. One second is equivalent to 300000 kilometers, which is artificially specified. Second, the speed of light is the only constant, and other physical quantities are based on it. For example, if we specify that the unit of mass 1 gram $\left(\mathrm{m}_{0}\right.$, mass of 1 cubic centimeter of water)is equivalent to the speed of light, formula 9 can be written as

$\mathrm{E}_{0}=\mathrm{m}_{0} \mathrm{c}^{2}$

Or

$\mathrm{E}_{0}=\mathrm{m}_{0}{ }^{3}=\mathrm{c}^{3}$

The formula 11 has two meanings. First, because 1 second is equivalent to 300000 kilometers, 1 second will not be equivalent to other lengths. Second Since 1 gram is equivalent to the speed of light, 1 gram is not equivalent to other speeds. Third, In formula 11, the energy of other 
substances is expressed in,

$\mathrm{E}_{1}=\mathrm{k}^{3}{ }_{1} \times \mathrm{m}_{0} \mathrm{c}^{2}$

$\mathrm{E}_{1}=\mathrm{k}^{3}{ }_{1} \times \mathrm{m}_{0}{ }^{3}=\mathrm{k}^{3}{ }_{1} \times \mathrm{c}^{3}$

Here $\mathrm{k}_{1}=\mathrm{m}_{1} / \mathrm{m}_{0}=\mathrm{v} / \mathrm{c}$

but the maximum energy value for $\mathrm{E}_{1}$ and coefficient $\mathrm{k}$ are the finite value close to infinity and cannot reach infinity, which is given by the definition of Theorem 1 .

\section{The meaning of acceleration force}

As mentioned earlier, the one quantity continuum representing infinity is artificially divided into limited parts. The time and space parts divided here can be compared in size, so this gives us the meaning of each physical quantity. In any case, these physical quantities do not have real meaning in infinity, and all these physical quantities only have meaning in finite range, and this finity is only artificially divided by us.

As mentioned above, all physical quantities are essentially space/time, that is, we cannot distinguish them by nature, which means that all physical quantities have both simple scalar and complex tensor concepts. For example, mass, a physical quantity, is usually a scalar in the physical sense, but from Theorem 1, it has the concept of tensor, so mass also has complex multidimensional curve and surface characteristics. For another example, the accelerated force is usually a tensor in the physical sense (5), but from Theorem 1, it also has a simple scalar concept. All the foregoing in this paper have been artificially selected by us as finite approximation, However, space and time are essentially infinite, and therefore, in a sense, the ultimate goal of man is to reach infinity. For example, can we leap to infinity by some means? Axiom 3 gives us no hope because the universe is a quantitative continuum, so we can neither increase nor decrease this infinite quantity. We can say that we are infinity ourself, but how can we change and leap to infinity? In theory, if we think of ourselves as a infinite quantity, then by the change of direction we can travel an infinitely distances and leap to infinity. This is a paradox because our goals stay within the bounds of our own limits. Our research on acceleration is based on this goal. In this sense, the ultimate goal of mankind is to obtain a specific and definite value nearer to the limit of each physical quantity (mainly the accelerating force), thus further approaching our ultimate goal, though it is impossible to achieve it.

\section{Reference}

1 Qing Li, The meaning of the infinitely great (preprint Authorea: DOI: 10.22541/au.160822935.50569408/v1)

2 Qing $\mathrm{Li}$.A geomerty consisting of singularities containing only integers.(preprint Research Square: DOI: 10.21203/rs.3.rs-219046/v1 )

3 Qing Li, The meaning of an infinitely great velocity. (unpublish data)

4 A. Einstein. "The Meaning of Relativity", Beijing Science Press. P28-30(1979)

5 The meaning of accelerated motion （preprint Zenodo: DOI:10.5281/zenodo.4448415) 
Author information:

Qing Li

\section{Affiliation:}

Department of Function, ShiJiaZhuang Traditional Chinese Medical Hospital Code Number: 050051

No. 233 ,ZhongShan West Road

ShiJiaZhuang City

HeBei Province

PR. China.

Tel.: +86-13833450232

E-mail: liqingliyang@126.com

Backup email: 2958773632@qq.com

Author contributions state: Qing Li does full work in this manuscript.

Funding Acknowledgement: This research received no specific grant from any funding agency

in the public, commercial, or not-for-profit sectors.

Conflict of interest statement: The authors declare no conflict of interest in preparing this article. 
